\title{
LOS RECURSOS EMPRESARIALES DE LAS PEQUEÑAS Y MEDIANAS EMPRESAS COMERCIALES (PYMES) DE LA CIUDAD DE AMBATO
}

\author{
Ana Lucia Quispe-Otacoma ${ }^{1}$, Mario Patricio Padilla-Martínez ${ }^{2}$
}

\begin{abstract}
The objective of the research was to propose the substitution of the technological infrastructure of small and medium-sized enterprises (SMEs) of Ambato city, in order to implement new automated systems, such as Enterprise Resource Planning (ERP), which facilitate the information concentration and decision making. Information and communication technologies were defined, because they constitute the innovative components that facilitate the work of human beings; and allow to perform reliable, efficacious, economical, ecological and ethical management. It was proven that the adaptation of a free software program ERP, as managerial tool, contributes to the entrepreneurial management of commercial SMEs.
\end{abstract}

Keywords: Entrepreneurial Management, ERP, SMEs, TICs.

CÓDIGO UNESCO: Administración 590900

\section{RESUMEN}

El objetivo de la investigación fue proponer la sustitución de la infraestructura tecnológica de las pequeñas y medianas empresas (PyMES) de la ciudad de Ambato, para implementar nuevos sistemas automatizados, como los ERP (Planificación de Recursos Empresariales, del inglés Enterprise Resource Planning), que faciliten la concentración de información y la toma de decisiones. Se definieron las tecnologías de la información y la comunicación, ya que estas constituyen los componentes innovadores que facilitan el trabajo del ser humano; y permiten realizar una gestión confiable, eficaz, económica, ecológica y ética. Se demostró que la adaptación de un software libre ERP, como herramienta gerencial, contribuye con la gestión empresarial de las PyMES comerciales.

Palabras clave: Gestión Empresarial, ERP, PyMES, TIC

\section{INTRODUCCIÓN}

A nivel internacional existe un ambiente competitivo en el campo empresarial, que requiere la promoción de los procesos y de las actividades de negocio, los cuales se consideran las ventajas competitivas de las empresas.

\footnotetext{
${ }^{1}$ Departamento de Ciencias Económicas, Administrativas y de Comercio, Universidad de las Fuerzas Armadas ESPE, Ecuador correo electrónico alquishpe3@espe.edu.ec

${ }^{2}$ Facultad de Ciencias Administrativas, Universidad Técnica de Ambato, Ecuador correo electrónico: mariopadillam@hotmail.com
} 
El deseo de sobresalir ante la competencia ha permitido que se le otorgue una mayor importancia a las tecnologías de la información y a su alineación con las estrategias corporativas, para mejorar sus procesos clave de negocio.

Una prueba de esto es el incremento sustancial de adquisiciones de paquetes de software empresariales, entre los que se encuentra el ERP (Planificación de Recursos Empresariales, del inglés Enterprise Resource Planning), mediante el cual los directivos esperan integrar las áreas, secciones o departamentos de la empresa que apoyan, para comercializar sus productos. No obstante, las empresas necesitan más herramientas que garanticen el control y la centralización de la información, con el fin de tomar las mejores decisiones con respecto a sus procesos y estrategias empresariales.

Los ERP constituyen un recurso significativo para aquellas empresas que buscan una solución universal a la centralización de información oportuna, veraz y precisa. La gestión empresarial necesita encontrar un camino hacia la excelencia, para desenvolverse en un mundo altamente competitivo, globalizado y tecnológico; puesto que el líder de la gestión también impulsa la obtención de resultados positivos, eficientes, eficaces, económicos, ecológicos y éticos, que beneficien a los clientes internos y externos.

La gestión empresarial -basada en la creatividad, la gestión tecnológica y la innovacióndebe estar presente en las organizaciones modernas de cualquier sector de la economía, sobre todo en pequeñas y medianas empresas (PyMES), las cuales se deben insertar en un mundo competitivo exigente. Dicha gestión se vincula con las tecnologías de la información y la comunicación (TIC), ya que estas permiten navegar en el mercado empresarial y contribuyen con el desarrollo económico-social del ambiente en el que se desarrollan los procesos de gestión. Las empresas, como unidades de producción, crean puestos de trabajo, impulsan y conforman la innovación, aceleran los cambios estructurales y aportan beneficios a la economía del país. En la mayoría de las empresas, es necesario remplazar la infraestructura tecnológica, e implementar nuevos sistemas automatizados que faciliten la concentración de información. Esto contribuiría con la toma de decisiones oportunas que coadyuven al crecimiento empresarial. Igualmente, se debería comprobar el nivel de capacitación de los involucrados en la generación de información, pues de este depende el buen desenvolvimiento del sistema.

Teniendo en cuenta lo planteado anteriormente, el objetivo de la investigación fue proponer la sustitución de la infraestructura tecnológica de las PyMES de la ciudad de Ambato, para implementar nuevos sistemas automatizados, como los ERP, que faciliten la concentración de información y la toma de decisiones.

\section{Métodos}

\section{Tecnologías de la información y la comunicación}


De acuerdo con lo señalado por Senn (2005), las TIC constituyen el medio por el que fluyen los datos de una persona o departamento hacia otros. Este flujo puede ser concentrado, desde la comunicación interna y las líneas telefónicas, hasta sistemas de cómputo que generan reportes para varios usuarios.

Según Laudon y Laudon (2012), las TIC son componentes interrelacionados que capturan, almacenan, procesan y distribuyen información, con el fin de apoyar la toma de decisiones, el control, el análisis y la visión institucional. Por tales motivos, las TIC se consideran una propuesta innovadora que contribuye con la gestión empresarial, mediante los ERP, y que permite controlar - de forma modular - la producción, la logística, la distribución, el inventario, los envíos, las facturas y la contabilidad.

Los objetivos principales de los sistemas ERP son:Optimizar los procesos empresariales.

- Acceder a la información.

- Compartir información entre los procesos empresariales.

- Eliminar datos y operaciones innecesarias.

Las diferentes marcas creadoras de software ERP (como SAP u Oracle) ofrecen beneficios característicos, que se deben tener en cuenta al adoptar la tecnología. Por otra parte, al optar por un software libre se debe considerar el campo de aplicación (en las PyMES) y la exigencia de los objetivos.

De acuerdo con lo planteado por el Ministerio de Industrias y Productividad (2012), un ERP es un sistema informático de administración gerencial que permite evaluar, controlar y gestionar, de manera más fácil y precisa, una empresa. Actualmente, dicho sistema constituye una necesidad, debido al aumento del nivel de información y a la influencia que esta ejerce en la toma de decisiones. En la figura 1 se muestra las características de un buen software ERP, según la Norma ISO 9126-1 (2005).

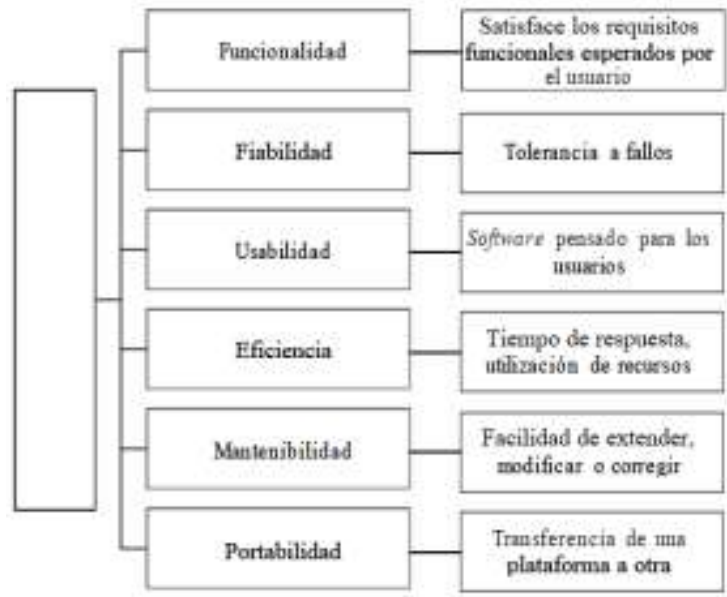

Figura 1. Caracteristicas de un buen sofhware ERP Fuente: Norma ISO 9126-1 (2005). 
La creación de un ERP, generalmente, resulta compleja, pues implica el rediseño de los esquemas de trabajo. Además, su implementación es riesgosa, debido al tamaño, los costos elevados y la inversión de tiempo. Por tales razones, se considera una alternativa la selección técnica de un software libre ERP que se adapte a los requerimientos empresariales, lo cual garantizaría la eficiencia de la gestión, así como beneficios a los clientes internos y externos.

El software libre permite que los usuarios ejecuten, copien, distribuyan, estudien, cambien y mejoren el software. Este se caracteriza por presentar las cuatro libertades siguientes:

- Libertad 0: ejecuta el programa sin restricciones.

- Libertad 1: permite el acceso al código fuente.

- Libertad 2: redistribuye copias con propósitos de ayuda.

- Libertad 3: distribuye versiones modificadas.

En la tabla 1 se muestra los softwares libres ERP para PyMES que existen en el mercado tecnológico.

Tabla 1. Softwares libres ERP para PyMES.

\begin{tabular}{lllll}
\hline ERP & Lenguaje Base & Licencia & Información & País \\
\hline Adempiere & Java & GPL & Antecesor Compiere & España \\
& JavaScript, & & & \\
ERPNEXT & MySQL & GPL & ERP para PYMES & India \\
HeliumV & Java & AGPL & ERP para PYMES & Austria y Alemania \\
OFBiz & Apache, Java & Apache & ERP para PYMES & Estados Unidos \\
& & License 2.0 & & \\
& & & & \\
\hline
\end{tabular}

Antes de seleccionar un ERP, es importante evaluar su funcionalidad, fiabilidad, usabilidad, eficiencia, mantenibilidad y portabilidad; sin embargo, resulta significativo considerar el costo y su flexibilidad para programar, estructurar y modular (tabla 2). 
Tabla 2. Comparación entre los diferentes ERP.

\begin{tabular}{|c|c|c|c|c|}
\hline ERP & Flexibilidad & Modular & Costo & Estatus \\
\hline ERPNEXT & Sí & Sí & Sí & - \\
\hline HeliumV & Sí & Sí & Sí & - \\
\hline OFBiz & Sí & Sí & Sí & - \\
\hline ADempiere & Sí & Sí & $\begin{array}{l}\text { Mínim } \\
\mathrm{o}\end{array}$ & $\begin{array}{l}\text { Tiene } 4 \\
\text { libertades }\end{array}$ \\
\hline
\end{tabular}

\section{Gestión empresarial}

La gestión empresarial es una actividad que realiza la persona que dirige una empresa o negocio, con el objetivo de mejorar su productividad y, por ende, su competitividad. Para que la gestión sea óptima, es necesario identificar los factores de los que depende el éxito de esta actividad, y tener en cuenta que se manejan recursos e información importante para la toma de decisiones (figura 2).

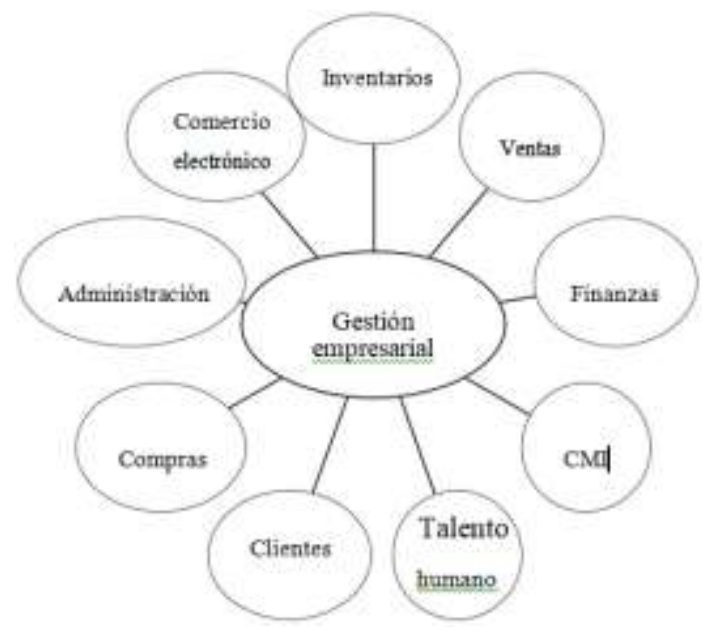

Figura 2. Información que requiere la gestion empresarial Fuente: elaboración propia.

- Inventarios. Abastecimiento continúo de los productos, considerando los inventarios óptimos, los tiempos de entrega, las estacionalidades, las demandas y 
las promociones. También se apoyan los costos y los tiempos favorables para las empresas.

- Ventas. Prometer fechas de entrega a las órdenes de venta y cumplirlas, velando por la sincronización de las compras, la distribución y la entrega, con el mejor aprovechamiento del talento humano.

- Finanzas. Aparato circulatorio de la empresa que se relaciona con el flujo de capital, los fondos que se posee para cumplir los objetivos de la organización, así como la manera de obtenerlos y gestionarlos.

- CMI (cuadro de mando integral). Proporciona un modelo de medición basado en indicadores (financieros y no financieros), cuya finalidad es mantener en alerta el cumplimiento del plan estratégico.

- Talento humano. Selección de personal idóneo, teniendo en cuenta las características del recurso humano que posee la empresa.

- Clientes. Administración de las relaciones que se establecen con los clientes. Permite conocer el comportamiento de estos, tener una proyección de las ventas y realizar el análisis histórico de las ventas por cliente.

- Compras. Eficiencia en el abastecimiento de productos, mediante la comparación de los precios, la calidad y las características de los proveedores. Esto permite seleccionar el más idóneo, lo que tiende a la disminución de los costos, al aumento de la eficiencia operativa y, como consecuencia, a obtener beneficios en la rentabilidad.

- Administración. Plan estratégico que direcciona el accionar de la empresa.

- Comercio electrónico. Uso de la Internet para hacer negocios. El diseño de la plataforma de negocios virtual debe considerar la seguridad, la accesibilidad y la practicidad.

\section{PyMES}

El tamaño de una empresa se puede catalogar, teniendo en cuenta varios elementos y en dependencia de la región. En el caso del Mercosur (Mercado Común del Sur), por ejemplo, se considera el número de empleados y el volumen de ventas. Por tanto, para que las PyMES sean clasificadas como tal, el número de empleados debe ser menor que 20 y el volumen de ventas no puede rebasar los 400000 dólares anuales.

Las PyMES son entes productivos o de servicios, que generan empleo y productividad en Ecuador y que permiten abastecer la demanda de productos y servicios de empresas nacionales, multinacionales e industrias, entre otras organizaciones. Para poder clasificarlas según su tamaño, se debe considerar el número de empleados efectivos (tabla 3), el valor bruto de las ventas anuales o el valor de activos totales. 
Tabla 3. Clasificación de las empresas, según número de empleados e ingresos por ventas brutas.

\begin{tabular}{lcccr}
\multicolumn{1}{c}{ Tamaño } & No. empleados & \multicolumn{2}{c}{$\begin{array}{c}\text { Ingreso por ventas } \\
\text { brutas }\end{array}$} \\
& Min. & Max. & Min.(USD) & Max.(USD) \\
\hline Microempresa & 1 & 9 & $<=$ & 100000 \\
Pequeña & 10 & 49 & 100001 & 1000000 \\
Mediana A & 50 & 99 & 1000001 & 2000000 \\
Mediana B & 100 & 199 & 2000001 & 5000000 \\
Grande & 200 & $>=$ & 5000001 & $>=$ \\
\hline
\end{tabular}

Fuente: Ecuador en cifras (2015) (http://www.ecuadorencifras.gob.ec/directoriodeempresas/).

En la tabla 4 se muestra las actividades económicas de las empresas registradas en Ecuador.

Tabla 4. Número de empresas por actividad económica.

\begin{tabular}{lrr}
\hline Actividad & \multicolumn{2}{c}{$\begin{array}{c}\text { Número de } \\
\text { empresas Porcentaje }\end{array}$} \\
\hline Comercio & 74566 & 38.97 \\
Agricultura & 3324 & 14.67 \\
Transporte & 59497 & 8.44 \\
Manufacturas & 52392 & 7.44 \\
Alojamiento & 51379 & 7.29 \\
Otros Servicios & 42040 & 5.97 \\
Técnico & 26804 & 3.80 \\
Construcción & 24729 & 3.51 \\
Inmobiliarias & 14114 & 2.00 \\
Salud & 13963 & 1.98 \\
Enseñanza & 11524 & 1.64 \\
Administrativos & 10696 & 1.52
\end{tabular}




$\begin{array}{lrr}\text { Información } & 6014 & 0.85 \\ \text { Artes } & 3753 & 0.53 \\ \text { Minas } & 3493 & 0.50 \\ \text { Administración } & & \\ \text { Pública } & 3359 & 0.48 \\ \text { Financieras } & 2320 & 0.33 \\ \text { Agua } & 338 & 0.05 \\ \text { Energía } & 251 & 0.04 \\ \text { Total } & 704556 & 100.00 \\ \end{array}$

Fuente: Instituto Nacional de Estadísticas y Censos (2010).

En Ecuador existen 704556 empresas, según el último Censo Nacional Económico de 2010, de las cuales $74566(38.97 \%)$ se dedican a la actividad económica del comercio. Estas se pueden considerar microempresas, pequeñas, medianas y grandes empresas (tabla 5).

Tabla 5. Empresas por actividad económica.

\begin{tabular}{lrrrr}
\hline \multirow{2}{*}{ Tamaño de la empresa } & \multicolumn{4}{c}{ Cantidad } \\
\cline { 2 - 5 } & \multicolumn{4}{c}{ Ecuador Porcentaje Tungurahua Porcentaje } \\
\hline Microempresas & 631430 & $89 \%$ & 30721 & $92 \%$ \\
Pequeñas empresas & 57772 & $8 \%$ & 2313 & $7 \%$ \\
Medianas empresas & 11797 & $2 \%$ & 411 & $1 \%$ \\
Grandes empresas & 3557 & $1 \%$ & 95 & $0 \%$ \\
Total & 704556 & $100 \%$ & 33540 & $100 \%$ \\
\hline
\end{tabular}

Fuente: Instituto Nacional de Estadísticas y Censos (2010).

Para el 2015 en el Ecuador se registran la información de empresas en una base de datos comprimida denominada bdd_empresas_2015 que contiene la base de datos empresa EMPRESAS_2015.sav a ser leída por el programa estadístico SPSS. Las variables 
estudiadas son: identificación, estratificación por actividad, estratificación por tamaño, estratificación por ubicación geográfica, variables demográficas y otras variables como clase de contribuyente de acuerdo con el Servicio de Rentas de Ecuador, y condiciones de operación (tabla 6).

\begin{tabular}{|c|c|c|}
\hline Variables & Indicador & Alternativa \\
\hline \multirow{14}{*}{$\begin{array}{l}\text { Identificación } \\
\text { (CAN, 2012) }\end{array}$} & Id_empresa & Número (11 dígitos) \\
\hline & & \\
\hline & Forma_institucional & 1. Rise \\
\hline & & $\begin{array}{l}\text { 2. Persona Natural no Obligada a llevar } \\
\text { Contabilidad }\end{array}$ \\
\hline & & $\begin{array}{l}\text { 3. Persona Natural Obligada a llevar } \\
\text { Contabilidad }\end{array}$ \\
\hline & & 4. Sociedad con fines lucro \\
\hline & & 5. Sociedad sin fines lucro \\
\hline & & 6. Empresa pública \\
\hline & & 7. Institución pública \\
\hline & & 8. Econ. Popular Solidaria \\
\hline & Unidad legal & 1. Persona Natural \\
\hline & & 2. Persona Jurídica \\
\hline & $\begin{array}{l}\text { Obligado a llevar } \\
\text { contabilidad }\end{array}$ & \\
\hline & & $\mathrm{S} \quad \mathrm{Si}$ \\
\hline \multirow[t]{2}{*}{ Estratificación } & Sector Económico & 1. Agricultura \\
\hline & & 2. Explotación minas y canteras \\
\hline \multirow[t]{2}{*}{ por } & & 3. Industria Manufacturera \\
\hline & & 4. Comercio \\
\hline \multirow[t]{5}{*}{ Actividad } & & 5. Construcción \\
\hline & & 6. Servicios \\
\hline & CIIU_sección & $\mathrm{A}, \mathrm{B}, \mathrm{C}, \ldots \mathrm{S}$ (19 secciones) \\
\hline & CIlU_división & Divisiones por cada sección (3) \\
\hline & CIIU_clase & Clases por división (hasta 4dig.) \\
\hline \multirow[t]{2}{*}{ Estratificación } & Número de empleados & 1. Estrato I $(<=9)$ \\
\hline & por estrato & 2. Estrato II (10-49) \\
\hline \multirow[t]{2}{*}{ por } & & 3. Estrato III (50-99) \\
\hline & & 4. Estrato IV (100-199) \\
\hline \multirow[t]{2}{*}{ Tamaño } & & 5. Estrato V $(>=200)$ \\
\hline & Número empleados & Número \\
\hline
\end{tabular}


Estrato de ventas

1. Estrato I $(<=100.000)$

2. Estrato II (100.001-1.000.000)

3. Estrato III (1.000.001-2.000.000)

4. Estrato IV (2.000.001-5.000.000)

5. Estrato V $(>=5.000 .001$

Tamaño empresa

1. Microempresa

2. Pequeña empresa

3. Mediana empresa A

4. Mediana empresa B

5. Grande empresa

Fuente: Ecuador en cifras (2015) (http://www.ecuadorencifras.gob.ec/directoriodeempresas/)

Tabla 6. Variables de estudio de las Empresas (continuación)

\begin{tabular}{|c|c|c|}
\hline Variables & Indicador & Alternativa \\
\hline \multirow[t]{4}{*}{$\begin{array}{l}\text { Ubicación } \\
\text { geográfica }\end{array}$} & Zona de planificación & $\begin{array}{l}\text { 1. Esmeraldas, Carchi, Imbabura, } \\
\text { Sucumbíos } \\
\text { 2. Pichincha, Napo y Orellana } \\
\text { 3. Cotopaxi, Tungurahua, Chimborazo } \\
\text { y Pastaza } \\
\text { 4. Manabí, Santo Domingo } \\
\text { 5. Santa Elena, Guayas, Los Ríos, } \\
\text { Bolívar y Galápagos } \\
\text { 6. Azuay, Cañar, Morona Santiago } \\
\text { 7. El Oro, Loja, Zamora Chinchipe } \\
\text { 8. Guayaquil, Durán, Samborondón } \\
\text { 9. Quito Distrito Metropolitano. }\end{array}$ \\
\hline & Código Provincia & 1 al 24 \\
\hline & Código cantón & Dos dígitos \\
\hline & Código parroquia & Dos dígitos \\
\hline \multirow{4}{*}{$\begin{array}{l}\text { Demográficas } \\
\text { (CEPAL,2013) }\end{array}$} & $\begin{array}{l}\text { Fecha inicio } \\
\text { actividades }\end{array}$ & aaaammdd \\
\hline & Fecha cese actividad & aaaammdd \\
\hline & $\begin{array}{l}\text { Fecha reinicio } \\
\text { actividad }\end{array}$ & aaaammdd \\
\hline & Fecha inscripción & aaaammdd \\
\hline
\end{tabular}


Fecha suspensión definitiva

Otras variables
Clase de contribuyente

(SRI. 2015) aaaammdd

ESP Contribuyente especial

RISE Rise

OTR Otro contribuyente

Condiciones de

1. Activo

Operación

Fuente: Ecuador en cifras (2015) (http://www.ecuadorencifras.gob.ec/directoriodeempresas/)

Tabla 7. Clasificación de las empresas de Ambato.

\begin{tabular}{lrr} 
Tamaño de la empresa & Ambato & Comerciales \\
\hline Microempresas & 18433 & 7189 \\
Pequeñas empresas & 1388 & 541 \\
Medianas empresas & 247 & 96 \\
Grandes empresas & 57 & 22 \\
Total & 20124 & 7848 \\
\hline
\end{tabular}

Fuente: Instituto Nacional de Estadísticas y Censos (2010).

Para el 2015 las 19 actividades se encuentran agrupadas en 6 sectores económicos: Primero Agricultura, ganadería, silvicultura y pesca; segundo explotación de minas y canteras; tercero industrias manufactureras; cuarto sector económico es comercio; quinto sector la construcción y el sexto sector es servicios. Los siguientes resultados cuantitativos de tamaño de empresa por sector está en la tabla No. 7.

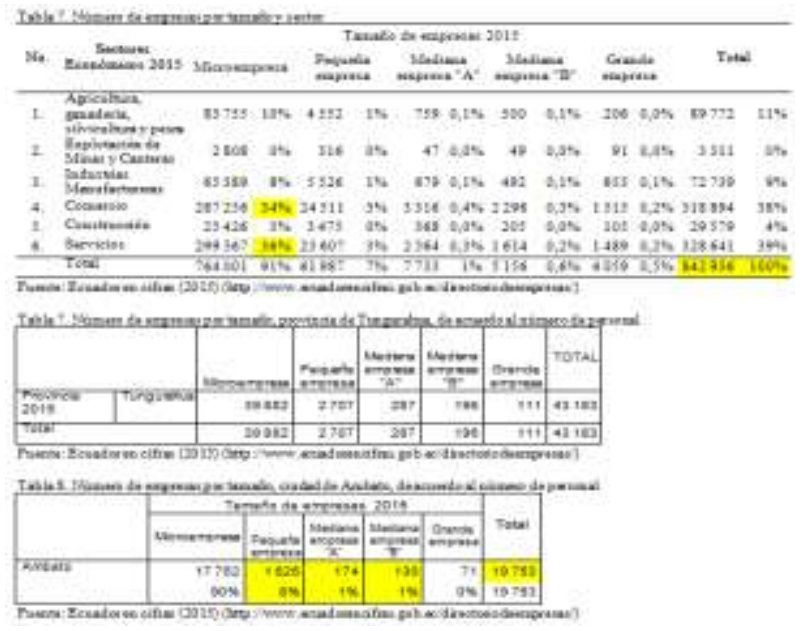




\section{Discusión}

De acuerdo con la investigación de campo, las PyMES de la ciudad de Ambato requieren que el ERP resuelva eficientemente la gestión de inventarios, las cuentas por pagar, las ventas, las compras, los informes personalizados, la contabilidad, la tesorería, la tributación, así como la información estratégica que debe recibir el gestor de forma rápida, oportuna y precisa. Las PyMES necesitan un ERP que gestione los recursos empresariales, al mejorar su estructura y complementar sus elementos, mediante la generación de un software que garantice su funcionalidad, fiabilidad, usabilidad, eficiencia, mantenibilidad y portabilidad.

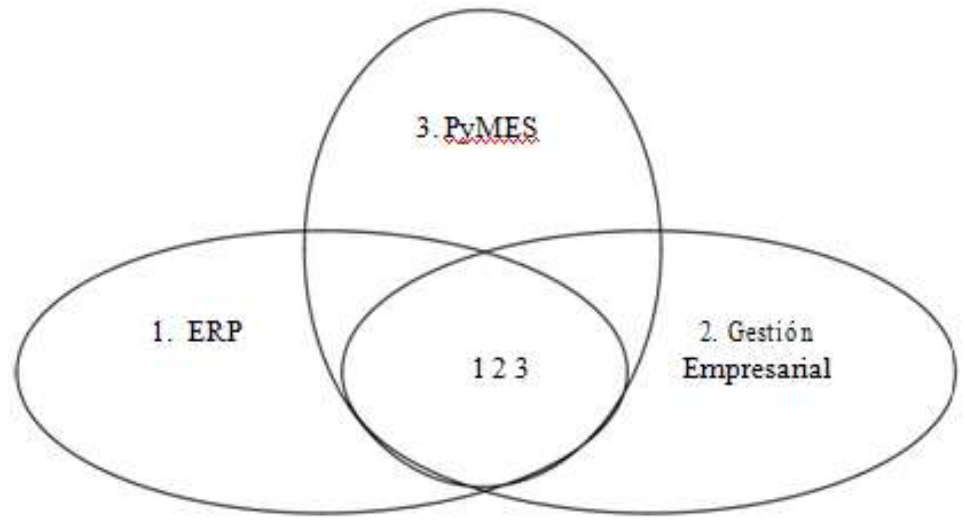

Figura 3. ERP123 para PyMES.

El ERP123 define un modelo de gestión empresarial, que abarca diferentes componentes interrelacionados (figura 4).

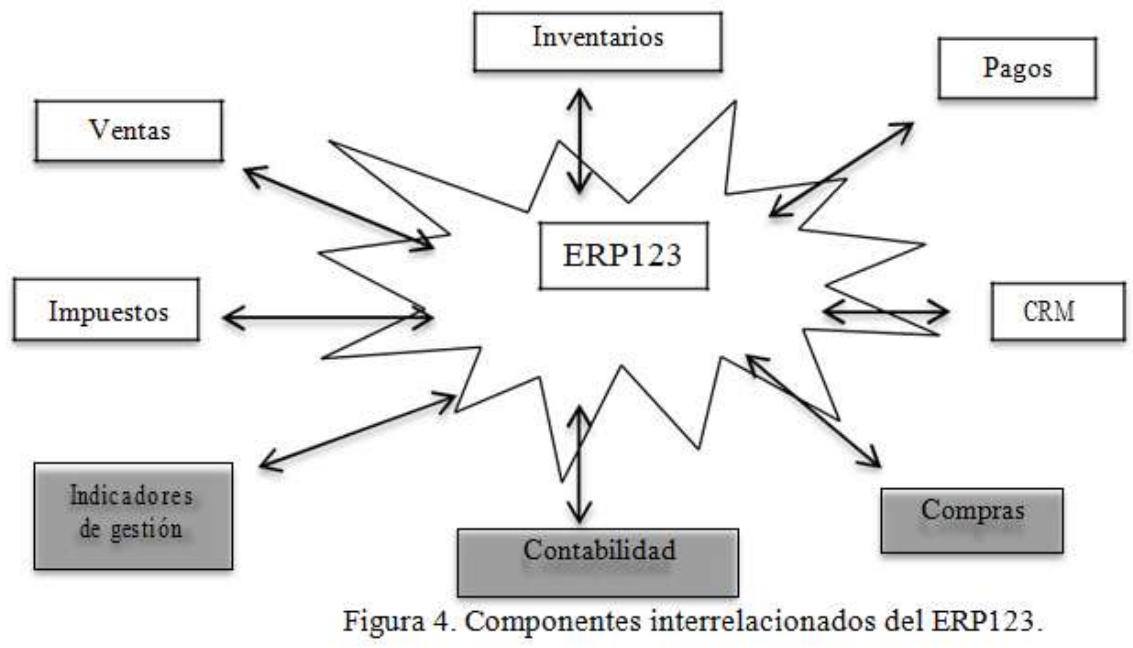




\section{Conclusiones}

1. A nivel local e internacional, existe un ambiente competitivo entre las empresas, fundamentalmente entre las PyMES, lo que exige la promoción de los procesos y las actividades de negocio para generar ventajas competitivas.

2. La mayor parte de las empresas de Ecuador son MiPyMES, por los que es preciso contar con un ERP para evaluar, controlar y gestionar los recursos.

3. Teniendo en cuenta el tamaño y los recursos financieros de las PyMES, es preferible utilizar un software libre ERP, como el ERP123.

\section{RECEIVED: JANUARY, 2017 \\ REVISED: MARCH, 2017}

\section{Referencias Bibliográficas}

Instituto Nacional de Estadísticas y Censos. Censo Nacional Económico, Ecuador. 2010

Disponible en: http://www.ecuadorencifras.gob.ec/censo-nacional-economico/ $[10 / 5 / 16]$.

ISO 9126-1. Estándar internacional para la evaluación de software, 2005.

Laudon, Kenneth C. y Laudon, Jane P. Sistemas de información gerencial. México: Pearson Educación, 2012. 643 p.

Ministerio de Industrias y Productividad (MIPRO). Sistema de Gestión de Recursos Empresariales, Ecuador, 2012. 60 p.

Senn, James A. Análisis y Diseño de Sistemas de Información. Segunda Edición. Mc Graw Hill, 2005.

Instituto Nacional de Estadísticas y Censos. Censo Nacional Económico, Ecuador. 2015 Disponible en: http://www.ecuadorencifras.gob.ec/directoriodeempresas/ [10/7/17].

Díaz Curbelo, Alina and Marrero Delgado, Fernando, «EL MODELO SCOR Y EL BALANCED SCORECARD, UNA PODEROSA COMBINACIÓN INTANGIBLE 
PARA LA GESTION EMPRESARIAL» Visión de Futuro, 2014, 18, 1, 36-57, 1668 8708.

Chusin Rosa, Cuchive Elvira, Guanotuña María Carmen, Iza Nancy, Maigua Luis, Moreno Martha, Morocho Dany, Nepas Julio, Pilatasig Erika, Robayo Esthela,Tipan Klever, Yánez Edison Talleres de capacitación para el fortalecimiento de los procesos financieros y contables de las Cajas Solidarias y Bancos Comunales de la Provincia de Cotopaxi.: Programa de Vinculación de la Universidad de las Fuerzas Armadas con la Sociedad Dra. Quispe Ana Lucia, 2015.

Laudon, Kenneth C. and Laudon, Jane P., Sistemas de información gerencial: Administración de la empresa digital, 8va., USA, Pearson Prentice Hall, 2006.

Martínez, Hugo and Perozo, Beatriz J., «Sistema de información gerencial para la optimización de portafolios de inversión» Revista Venezolana de Gerencia, 2010, 15, $50,253-272,1315-9984$.

Bravo Carrasco, Juan, Gestión por procesos en Chile 2012. Diagnóstico \& Propuestas, Santiago de Chile, Evolución S.A., 2012, 978-956-7604-22-7.

Blanco Encinosa, Lázaro J, La informática en la dirección de empresas, 1ra, La Habana, Félix Varela, 2011, 978-959-07-1629-4.

Noguera Hidalgo, Ángela Lucía[et al.], «Estrategia organizacional: una propuesta de estudio» Estudios Gerenciales, 2014, 30, 131, 153-161, 0123-5923.

González, Virginia[et al.], «Implementación de un Sistema de Control de Gestión con las Metodologías del Balanced Scorecard y Gestión Por Procesos en una Pymes Comercial en la ciudad de Guayaquil durante el año 2007»?,

Ruiz-Fuentes, Daysi[et al.], «La gestión por procesos, su surgimiento y aspectos teóricos» Ciencias Holguín, 2014, 20, 1, 10-19, 1027-2127.

Organización Internacional de Normalización (ISO), «NORMA INTERNACIONAL ISO 9001:2008»2008,

Medina León, A[et al.], «La documentación de procesos y su formalización, herramienta para la integración de los sistemas de gestión y la sustentabilidad», Organizaciones Sustentables, Veracruz, México, Universidad Veracruzana, 2014, 978-607-9248-63-5. 
Fernández-Morales, Mynor, «Control estratégico de gestión en unidades de información» ECiencias de la Información, 2014, 4, 1, 1659-4142.

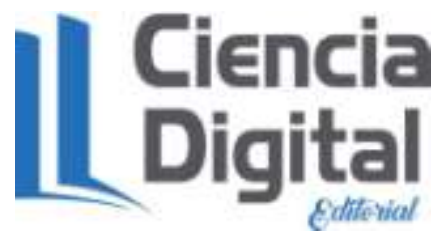

El artículo que se publica es de exclusiva responsabilidad de los autores y no necesariamente reflejan el pensamiento de la Revista Ciencia Digital.

El articulo queda en propiedad de la revista y, por tanto, su publicación parcial y/o total en otro medio tiene que ser autorizado por el director de la Revista Ciencia
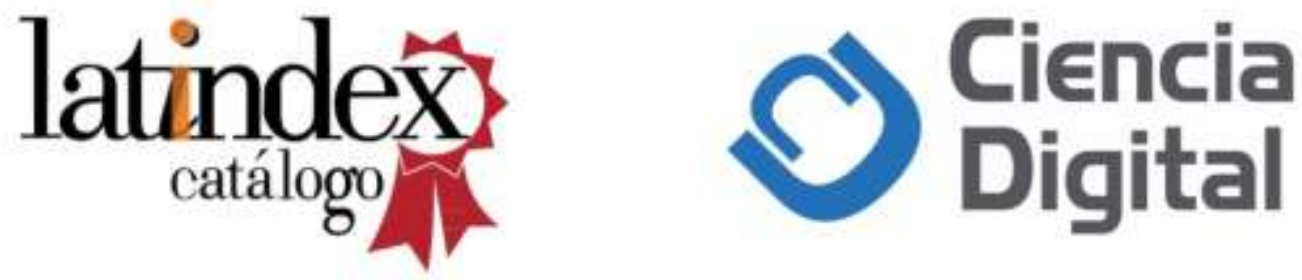